\title{
Modellierung und Simulation am Lehrstuhl für Thermoprozesstechnik
}

\author{
Christoph Spijker, Andreas Rath und Harald Raupenstrauch \\ Lehrstuhl für Thermoprozesstechnik, Montanuniversität Leoben, Leoben, Österreich \\ Eingegangen 30. April 2020; angenommen 28. Mai 2020; online publiziert 16. Juni 2020
}

Zusammenfassung: Die Arbeitsgruppe Modellierung und Simulation am Lehrstuhl für Thermoprozesstechnik beschäftigt sich primär mit der Modellierung von Verbrennungsprozessen, wobei diese Modelle meist auf industrielle Öfen angewendet werden. Je nach Aufgabenstellung wurden spezifische Modelle entwickelt, um die jeweiligen Aggregate abzubilden. Besonders zu erwähnen sind die NOx Postprocessoren, welche eine präzise Vorhersage der Stickoxidkonzentrationen mit geringem Rechenaufwand ermöglichen. Neben der numerischen Strömungsmodellierung befasst sich die Arbeitsgruppe mit dem Blick Richtung Industrie $4.0 \mathrm{im}$ Projekt FalstCalc mit Multieindimensionalen Ofenmodellen, welche mehrere Tage einer Ofenreise in Minuten abbilden können.

Schlüsselwörter: Modellierung, Verbrennung, Ofenanlagen

Modeling and Simulation at the Chair of Thermal Processing Technology

Abstract: The work group modeling and simulation at the Chair of Thermal Processing Technology is primarily concerned with modeling combustion processes, whereby these models are mostly applied to industrial furnaces. Over the years a wide variety of computational methods has been developed. These are mostly used to model industrial furnaces. One highlight of these methods are the NOx postprocessors, which allow a precise prediction of nitrogen oxides formation with reusable calculation effort so that these models are applicable to industrial furnaces. The transformation towards industry 4.0 requires fast calculating models. To fulfill the demand, the multi-onedimensional approach called "FastCalc" was created. This

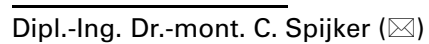

Lehrstuhl für Thermoprozesstechnik,

Montanuniversität Leoben,

Franz Josef-Straße 18,

8700 Leoben, Österreich

christoph.spijker@unileoben.ac.at approach allows to predict multiple days of the behavior within a furnace in minutes of calculation time.

Keywords: Modeling, CFD, Combustion, Industrial furnaces

\section{Einleitung}

Die Arbeitsgruppe "Modellierung und Simulation“ am Lehrstuhl für Thermoprozesstechnik der Montanuniversität Leoben befasst sich primär mit der Computational Fluid Dynamics (CFD) Modellierung von Verbrennungsprozessen, wobei die Modellierung von industriellen Ofenanlagen das größte Anwendungsgebiet abbildet. Durch stetig neue Aufgabenstellungen wurden neue Modelle entwickelt, die das Knowhow in den verschiedensten technischen Arbeitsbereichen, wie etwa der Brennstoff- und Verbrennungstechnik, der Steigerung der Recheneffizienz bis hin zur Sicherheitstechnik erweitert haben. So befasst sich der Lehrstuhl nicht nur mit der Modellierung von industriellen Öfen und Brennern, sondern auch mit der Modellierung von Versuchsanlagen, wie dem Flash-Reaktor oder dem induktiv beheizen Koksbett der InduRed Anlage. Durch die Tätigkeiten der Arbeitsgruppe „Prozess- und Anlagensicherheit" am Lehrstuhl entstand auch das Themenfeld der Modellierung von Gas- und Staubexplosionen sowie Selbsterwärmungsprozesse von Schüttgütern. Aufgrund der Wandelung Richtung Industrie 4.0 beschäftigt sich die Arbeitsgruppe "Modellierung und Simulation“ auch mit schnell rechnenden multieindimensionalen Ofenmodellen im Projekt FastCalc. Diese können eine Ofenreise von mehreren Tagen in wenigen Minuten abbilden. Im Rahmen dieser Veröffentlichung werden zwei Entwicklungen des Lehrstuhls bezüglich Recheneffizienz besonders hervorgehoben, zum einen die Modellierung von Stickoxiden, zum anderen das Projekt FastCalc. 


\section{Modellierung von Ofenanlagen}

Der Fokus bei der Modellierung von Ofenanlagen liegt auf der Verbesserung des Verständnisses der im Ofen vorherrschenden Prozessparameter und deren Interaktionen zueinander. Mit diesem Prozessverständnis können einerseits Maßnahmen abgeleitet und deren Auswirkungen andererseits durch das Modell überprüft werden. Da sich industrielle Ofenanlagen in ihrer Bauweise unterscheiden, werden für diese jeweils eine Kombination individueller Submodelle eingesetzt. Für gasbefeuerte Öfen mit Mündungsmischbrennern kommt hier meist das am Lehrstuhl entwickelte Flamelet-Equilibrium-Hybrid Modell [1] zum Einsatz, welches eine präzise Vorhersage der Temperaturen und chemischen Zusammensetzung der Ofenatmosphäre mit mäBigem Rechenaufwand ermöglicht. Für vorgemischte Brenner sowie flammlose Verbrennungen werden Mischungsund kinetikkontrollierte Verbrennungsmodelle eingesetzt, wobei anwendungsabhängig zwischen dem Eddy Dissipation Concept (EDC) [2] oder dem Partially Steered Reactor (PaRS) [3] Modell gewählt wird. Diese Modelle kommen ebenfalls bei der Modellierung heterogener Verbrennung in Kombination mit Partikelmodellen, welche die Trocknung, Pyrolyse und Koksausbrand beschreiben, zum Einsatz. Um den Transport des Brennguts zu modellieren, stehen auch eine Reihe von Modellen zur Verfügung. Für Stahlbänder kommt das am Lehrstuhl entwickelte Pseudo-Fluid [4] Modell zum Einsatz, welches den Vorteil bietet, einen beliebigen Verlauf eines Stahlbands, wie etwa die Umlenkung durch Ofenrollen, abzubilden (Abb. 1). Dieses Modell kann aber auch für den Transport in Hubbalken- oder Stoßöfen eingesetzt werden.

Zur Beschreibung bewegter Schüttungen wurde von Pollhammer [5] am Lehrstuhl ein Diskretes Elemente Modell (DEM) entwickelt, welches mit einem CFD Modell gekoppelt ist. Dieses Modell ist in der Lage Partikel-Par-

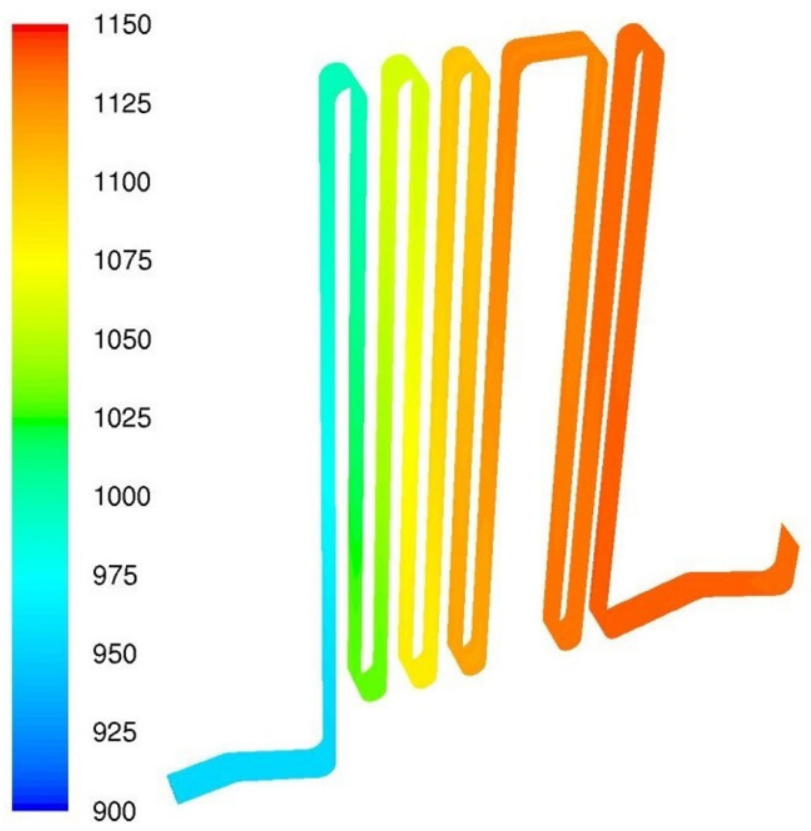

Abb. 1: Temperaturprofil [K] eines Stahlbands in einem Glühofen [4]

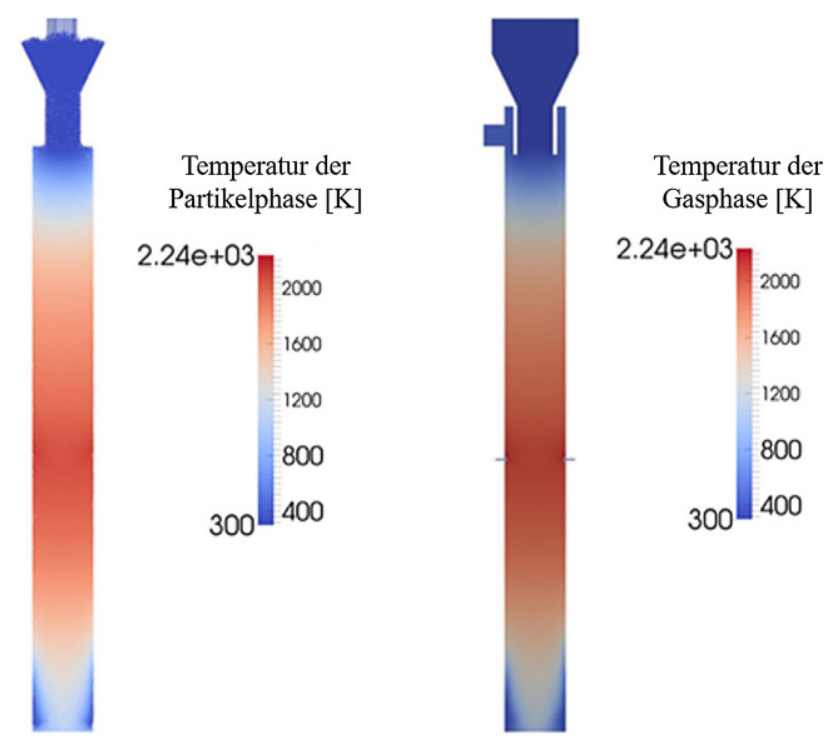

Abb. 2: Abbildung der Temperatur der Partikel und Gasphase in einem Schachtofen [5]

tikel Wärmeleitung sowie Partikel-Partikel Strahlung zu beschreiben und kann mit einem beliebigen Verbrennungsmodell gekoppelt werden. Die Anwendung dieses Modells auf einen Schachtofen ist in Abb. 2 dargestellt.

Für die Modellierung des Wärme- und Stofftransports zwischen der Ofenatmosphäre und des Brenngutes der jeweiligen Ofenmodelle kann wieder auf verschiedenste am Lehrstuhl entwickelte Submodelle zurückgegriffen werden. Hier können Modelle zur Zunderbildung, Oxidation, Reduktion und gezielter Entkohlung hervorgehoben werden, die simulationsparametergesteuert den akkuraten Wärmetransport beschreiben.

\section{Stickoxidmodellierung}

Durch die stetig steigenden Anforderungen hinsichtlich Emissionen von industriellen Ofenanlagen wurde es notwendig, eigene Wege im Bereich der Modellierung von Stickoxiden zu beschreiten. Mischungs- und kinetikkontrollierte Verbrennungsmodelle, wie beispielsweise das Eddy Dissipation Concept (EDC) [2], sind in Kombination mit komplexen Reaktionsmechanismen in der Lage, die Bildung von Stickoxiden korrekt abzubilden. Diese Modelle sind jedoch so rechenintensiv, dass diese für industrielle Anwendungen faktisch nicht anwendbar sind. Auf dem Flamelet Ansatz [6] basierende Modelle, wie das am Lehrstuhl entwickelte Flamelet-Equilibrium-Hybrid Modell [1], beruhen auf der Annahme einer dünnen Flammenfront. Die Bildung von Stickoxiden beinhaltet kinetisch langsame Reaktionen, welche dieser Annahme nicht gerecht werden. Jedoch bilden diese Modelle Temperatur, Strömung, Turbulenz und die makroskopischen Spezies präzise ab. Beim am Lehrstuhl entwickeltem Ansatz handelt es sich um ein Postprocessing, bei dem auf Basis konstanter Temperatur-, Strömungs- und Turbulenzfelder die chemischen Reaktionen neu gelöst werden. Der erste Ansatz (Version 1+) 
Abb. 3: Kontur des Massenbruchs an NO der Sandia Flamme D, modelliert mit dem Postprocessor der Version 2

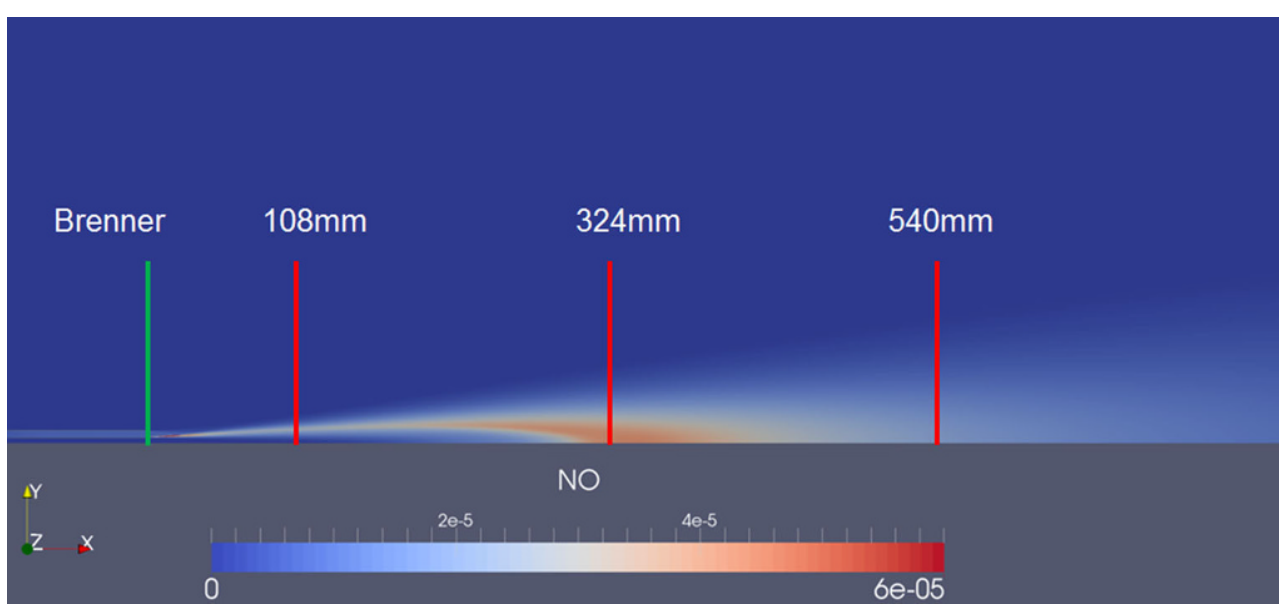

verwendet den einfachen thermischen $\mathrm{NO}$ Mechanismus nach Hanson [7] und betrachtet alle anderen chemischen Spezies als konstant. Aufgrund der geringen Komplexität des Mechanismus benötigt das Postprocessing einer Ofenanlage nur einige Stunden. Dieser Postprocessor ist in der Lage, die Bildungszonen qualitativ vorherzusagen. Dies ist jedoch für eine Optimierung von Brennern und Ofenanlagen oft ausreichend.

Um auch Stickoxide quantitativ zu modellieren, wurde die Version 2 des Postprocessors entwickelt. Diese löst auf Basis komplexer Reaktionsmechanismen die Felder für die chemischen Spezies neu. Da dieser Prozess ebenfalls zu rechenintensiv für industrielle Anwendungen ist, wurde zum Lösen der Reaktionen ein Filterverfahren entwickelt, welches die für die Stickoxidbildung relevanten Rechenzellen ermittelt und nur in diesen die Reaktionen löst. Dieses Verfahren reduziert die Rechenzeit um $85 \%$. In Abb. 3 ist der modellierte Massenbruch an NO der Sandia Flamme D dargestellt. Die Verläufe des Massenbruchs in den hier angeführten Schnitten werden in Abb. 4 wiedergegeben. Hierbei ist ersichtlich, dass die modellierten Konzentrationen des
Postprocessors sehr gut mit den Messungen von Barlow [8] korrelieren.

Der Postprocessor der Version 2 wird zur Version 2.0+ weiterentwickelt, um die Rechenzeit noch weiter zu senken. Diese verfolgt drei weitere Ansätze. Zum einen wird der innere Aufbau des Modells optimiert, um die Zahl der Rechenoperationen zu senken. Dies reduziert die Rechenzeit um $35 \%$. Des Weiteren wurde auf Basis der Version 1 den Postprocessors eine Anfangswertkorrektur implementiert. Durch die besseren Anfangsbedingungen reduziert sich der Rechenaufwand um weitere $32 \%$. Der dritte Ansatz befindet sich derzeit in der Testphase. Hier werden auf Basis vorhandener Ergebnisse eigene Rechengitter für den Postprocessor generiert. Erste Tests an Referenzflammen zeigen eine Reduktion der Zellanzahl um ca. 52\%.

\section{Multieindimensionale Ofenmodelle- FastCalc}

Im Projekt FastCalc wird das Potential von vereinfachten Ofenmodellen zur Unterstützung von kurzfristigen Prozess-
Abb. 4: Gegenüberstellung des Massenbruchs an NO zwischen dem Postprocessor der Version 2 und Messungen [8] der Sandia Flamme D. Die Position der Schnitte kann Abb. 3 entnommen werden

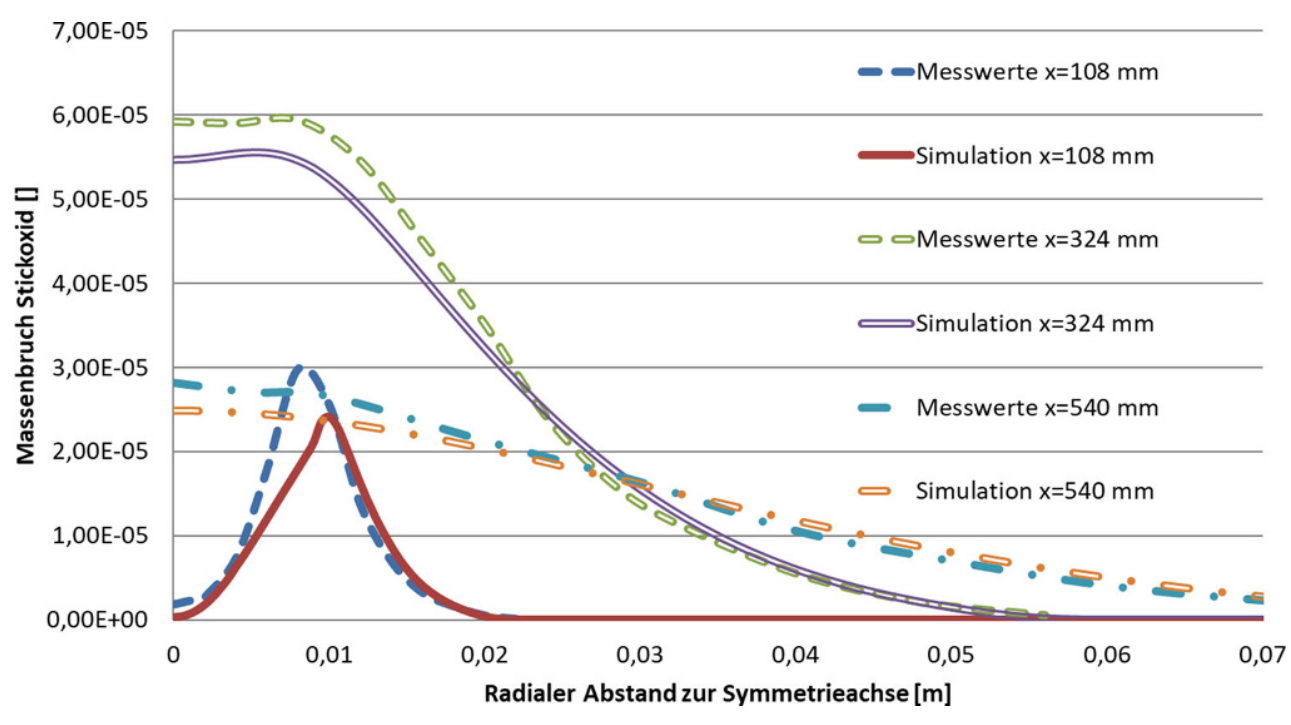


und Managemententscheidungen untersucht. Ziel des Projektes ist es, eine simulationsbasierte, effiziente Alternative zu anderen Datengenerierungsmechanismen, wie etwa CFD-Simulationen bzw. Experimenten zu etablieren, da diese oft zeitaufwändig sind. Dabei werden verschiedene Ansätze hinsichtlich der Modellaufteilung und geometrischer Diskretisierung analysiert und ein Optimum zwischen Vereinfachung und Rechenzeit gewählt. Grundsätzlich bedient sich das Konzept einer impliziten, multiplen und eindimensionalen finiten Volumen-Diskretisierungsmethode, wobei ein normalerweise benötigtes dreidimensionales Gitter in ineinander gekoppelte eindimensionale Zonen eingeteilt wird. Durch diesen Schritt verringert sich die benötigte Zellanzahl und die damit verbundene Anzahl der zu lösenden Gleichungen drastisch, wodurch eine signifikante Beschleunigung bezüglich der Rechenzeit erreicht wird. Allgemein lässt sich sagen, dass sich durch die starke Vereinfachung innerhalb weniger Minuten Rechenzeit mehrere Tage an Produktionszeit simulieren lassen. Die dabei generierten Daten sind aber als global gemittelt zu betrachten und lassen keine Rückschlüsse auf lokale Effekte zu. Aus diesem Grund dient FastCalc als unterstützendes Simulationstool, mit dem globale Parameter, wie etwa die durchschnittliche Ofenraumtemperatur, prognostiziert werden können und somit eine Aussage über den allgemeinen Prozessablauf erlaubt. In Zukunft soll es ermöglicht werden, gewünschte Prozessabläufe als Inputparameter zur Verfügung zu stellen und aus diesen die benötigten Kontrollparameter des Ofens zu berechnen. Dies würde eine optimale Grundlage für eine automatisierte Prozesssteuerung bieten, da sich alle Betriebsparameter aus Vorgaben berechnen lassen und somit keine Messungen and Produkt oder Ofen vorgenommen werden müssen.

Funding. Open access funding provided by Montanuniversität Leoben.

Open Access Dieser Artikel wird unter der Creative Commons Namensnennung 4.0 International Lizenz veröffentlicht, welche die Nutzung, Vervielfältigung, Bearbeitung, Verbreitung und Wiedergabe in jeglichem Medium und Format erlaubt, sofern Sie den/die ursprünglichen Autor(en) und die Quelle ordnungsgemäß nennen, einen Link zur Creative
Commons Lizenz beifügen und angeben, ob Änderungen vorgenommen wurden.

Die in diesem Artikel enthaltenen Bilder und sonstiges Drittmaterial unterliegen ebenfalls der genannten Creative Commons Lizenz, sofern sich aus der Abbildungslegende nichts anderes ergibt. Sofern das betreffende Material nicht unter der genannten Creative Commons Lizenz steht und die betreffende Handlung nicht nach gesetzlichen Vorschriften erlaubt ist, ist für die oben aufgeführten Weiterverwendungen des Materials die Einwilligung des jeweiligen Rechteinhabers einzuholen.

Weitere Details zur Lizenz entnehmen Sie bitte der Lizenzinformation auf http://creativecommons.org/licenses/by/4.0/deed.de.

\section{Literatur}

1. Edler, F.; Spijker, C.; Pollhammer, W.; Raonic, Z.; Raupenstrauch, H.: Modeling of a direct fired reduction reactor with a flamelet equilibrium hybrid model including transient reactive particles for design studies, 17th International Conference on Numerical Combustion, Book of Abstracts, 2019

2. Magnussen, B.: On the structure of turbulence and a generalized eddy dissipation concept for chemical reaction in turbulent flow, 19th Aerospace Sciences Meeting (1981), St. Louis, MO, USA, 1981

3. Kärrholm, F. P.: Numerical modelling of diesel spray injection, turbulence interaction and combustion, PhD Thesis, Göteborg: Chalmers University, 2008

4. Triebl, C.; Spijker, C.; Raupenstrauch, H.; Jarosik, A.; Angeli, G.: Modeling of the Annealing Furnace in a Hot-dip Galvanizing Line, INFUB-10th European Conference on Industrial Furnaces and Boilers, 2015

5. Pollhammer, W. R.: A cfd-dem model for nitrogen oxide prediction in shaft furnaces using openFOAM, PhD Thesis, Montanuniversitaet Leoben, 2019

6. Peters, N.: Laminar diffusion flamelet models in non-premixed turbulent combustion, Progress in energy and combustion science, 10, 198, pp 319-339

7. Hanson, R.; Salimian, K.: Survey of rate constants in the $\mathrm{N} / \mathrm{H} / \mathrm{O}$ system, Combustion chemistry, New York, NY: Springer, 1984, S. $361-421$

8. Barlow, R.; Frank, J.: Piloted ch4/air flames c, d, e, and f-release 2.1, Livermore USA, 2007

Hinweis des Verlags. Der Verlag bleibt in Hinblick auf geografische Zuordnungen und Gebietsbezeichnungen in veröffentlichten Karten und Institutsadressen neutral. 